\title{
Functional Analysis of a Higher Olfactory Center, the Lateral Horn
}

\author{
Nitin Gupta and Mark Stopfer \\ National Institute of Child Health and Human Development, National Institutes of Health, Bethesda, Maryland 20892
}

The lateral horn (LH) of the insect brain is thought to play several important roles in olfaction, including maintaining the sparseness of responses to odors by means of feedforward inhibition, and encoding preferences for innately meaningful odors. Yet relatively little is known of the structure and function of LH neurons (LHNs), making it difficult to evaluate these ideas. Here we surveyed $>250$ LHNs in locusts using intracellular recordings to characterize their responses to sensory stimuli, dye-fills to characterize their morphologies, and immunostaining to characterize their neurotransmitters. We found a great diversity of LHNs, suggesting this area may play multiple roles. Yet, surprisingly, we found no evidence to support a role for these neurons in the feedforward inhibition proposed to mediate olfactory response sparsening; instead, it appears that another mechanism, feedback inhibition from the giant GABAergic neuron, serves this function. Further, all LHNs we observed responded to all odors we tested, making it unlikely these LHNs serve as labeled lines mediating specific behavioral responses to specific odors. Our results rather point to three other possible roles of LHNs: extracting general stimulus features such as odor intensity; mediating bilateral integration of sensory information; and integrating multimodal sensory stimuli.

\section{Introduction}

The insect olfactory system has been useful for exploring sensory coding and plasticity (Hansson, 1999; Laurent, 2002; Carey and Carlson, 2011). In the locust, olfactory information is sent from peripheral olfactory organs to the antennal lobe (AL) and is then carried as the oscillatory output of a synchronized population of projection neurons (PNs) (Wehr and Laurent, 1996) to two higher olfactory centers. One center, the mushroom body (MB), has been studied extensively and is thought to be a site for olfactory learning (Davis, 2011). The other center, the lateral horn (LH), an area defined by the terminal arborizations of PNs in the lateral protocerebrum (Ernst et al., 1977; Homberg et al., 1989), remains poorly understood. What roles might the LH play in olfaction? Two hypotheses are prominent.

Perez-Orive et al. (2002) focused on a population of interneurons in the LH [LHIs, a subtype of LH neurons (LHNs)] that extends processes to the $\mathrm{MB}$ and appears to be GABAergic. Kenyon cells (KCs) in the MB receive oscillatory waves of excitatory input directly from PNs; Perez-Orive et al. (2002) proposed the LHIs, which also receive this excitatory input from PNs, then

\footnotetext{
Received March 4, 2012; revised April 4, 2012; accepted April 6, 2012.

Author contributions: N.G. and M.S. designed research; N.G. performed research; N.G. analyzed data; N.G. and M.S. wrote the paper.

This work was supported by an intramural grant from the National Institute of Child Health and Human Development (NICHD), National Institutes of Health (M.S.). Microscopy was performed at the Microscopy \& Imaging Core (NICHD) with the kind assistance of Vincent Schram and Louis Dye. We thank Sarah Kim and Joby Joseph for their help in developing the project; Diana Cummings and Leonardo Belluscio for antibodies; Collins Assisi, Maxim Bazhenov, Evan Butler, Paolo Forni, Sachiko Murase, Annalisa Scimemi, and members of the Stopfer laboratory for helpful discussions. We also thank Kui Sun for excellent animal care.

Correspondence should be addressed to Mark Stopfer, National Institute of Child Health and Human Development, National Institutes of Health, 35 Lincoln Drive, Bethesda, MD 20892. E-mail: stopferm@mail.nih.gov.

DOI:10.1523/JNEUROSCI.1066-12.2012

Copyright $\odot 2012$ the authors $\quad 0270-6474 / 12 / 328138-11 \$ 15.00 / 0$
}

feed it forward, after a brief delay, as inhibition to KCs. KCs would thus receive alternating waves of excitation from PNs and inhibition from LHIs, which together define temporal integration windows that help maintain the minimal and specific odorelicited spiking observed in KCs.

The LH has also been proposed to encode innate olfactory preferences. Abolishing the MB in Drosophila was linked to deficits in olfactory learning but had little effect on innate olfactory behaviors (de Belle and Heisenberg, 1994; Kido and Ito, 2002). Further, reducing olfactory input to both the $\mathrm{MB}$ and the $\mathrm{LH}$ caused deficits in innate behaviors, leading Heimbeck et al. (2001) to argue, by exclusion, that the LH could mediate innate, unlearned responses. Consistent with this proposal, anatomical studies in Drosophila showed stereotypical clustering of PN arborizations in the LH (Marin et al., 2002; Wong et al., 2002; Tanaka et al., 2004). Further, the LH appears to provide labeled lines for processing pheromones and mediating sexual behaviors (Jefferis et al., 2007; Ruta et al., 2010). However, whether the LH provides specialized pathways for specific odors processed by the general (nonpheromonal) olfactory system remains unclear because LHNs could integrate input from diverse PNs (Jefferis et al., 2007) and blocking synaptic transmission in the MB can cause deficits in innate behaviors (Wang et al., 2003; Xia and Tully, 2007).

Information about the morphology and responses of LHNs is needed to test these ideas. Here, we examined the structure and functions of the LH with an anatomical and electrophysiological survey in the locust. Our results are inconsistent with widely accepted views on the composition of the $\mathrm{LH}$, on the mechanisms underlying the sparseness of odor representations and the decoding of the PN input in KCs, and on the role of different LHNs in encoding innate preferences. Our results rather suggest the $\mathrm{LH}$ 
may contribute to concentration coding, bilateral processing, and multimodal integration.

\section{Materials and Methods}

Stimulus delivery. Odors were delivered as described previously (Brown et al., 2005). Briefly, $20 \mathrm{ml}$ of odorant solutions were placed in $60 \mathrm{ml}$ glass bottles at dilutions of $10 \%$ or $0.1 \%(\mathrm{v} / \mathrm{v})$ in mineral oil. Odorants used in our study were as follows: grass volatiles hexanol (hex), octanol (oct), hexanal (hxa), and 1-octen-3-ol (o3l); the synthetic compound cyclohexanone (chx); and nongrass plant volatiles geraniol (ger), citral (cit), and methyl jasmonate (mja), an oviposition deterrent (Daly et al., 2001). Odor pulses were delivered by puffing a measured volume $(\sim 0.1 \mathrm{~L} / \mathrm{min})$ of the static headspace above the odorants into an activated carbon-filtered air stream $(\sim 0.75$ $\mathrm{L} / \mathrm{min}$ ) flowing continuously across the antenna. A large $(11 \mathrm{~cm})$ vacuum funnel placed behind the antenna quickly removed the odorants. In some experiments, a bright white LED (7000 millicandela; RadioShack) placed $10 \mathrm{~cm}$ from the eyes and a piezo pulse buzzer (RadioShack) placed $50 \mathrm{~cm}$ from the animal provided visual and auditory stimuli, respectively.

Electrophysiology. Recordings were made from 102 adult locusts (Schistocerca americana) of either sex raised in our crowded colony. Insects were immobilized, and their brains were exposed, desheathed, and superfused with locust saline at room temperature as described previously (Stopfer and Laurent, 1999). Local field potential (LFP) recordings were made in the MB calyx using blunt glass micropipettes (6-15 M $\Omega$ when filled with saline) pulled by a horizontal puller (P87; Sutter Instrument). Intracellular recordings were made using sharp glass micropipettes $(60-200 \mathrm{M} \Omega$, when filled with $0.5 \mathrm{M}$ potassium acetate or an intracellular dye solution). The signals were amplified in bridge mode (Axoclamp-2B; Molecular Devices), further amplified with a DC amplifier (Brownlee Precision), and sampled at $15 \mathrm{kHz}$ (LabView software; PCI-MIO-16E-4 DAQ cards; National Instruments). After recording, dyes were injected into the neurons iontophoretically using $0.2-2 \mathrm{nA}$ current pulses at $3 \mathrm{~Hz}$ for up to $20 \mathrm{~min}$.

Histology and immunostaining. The intracellular dye solution consisted of $1 \%$ Neurobiotin (Vector Laboratories) in $0.2 \mathrm{M} \mathrm{LiCl}$ or $5 \%$ Lucifer yellow, Alexa Fluor 568, or Alexa Fluor 633 (Invitrogen) filled at the tip with $0.2 \mathrm{M} \mathrm{LiCl}$ in the shaft. Histological steps and confocal imaging were performed as described previously (Ito et al., 2008). Anti-GABA immunostaining was performed on whole locust brains containing dyefilled neurons using the procedure of Perez-Orive et al. (2002). Briefly, brains were taken from the glass slides and gradually hydrated through a series of $100,90,80,70,50$, and $30 \%$ ethanol baths, and were washed with PBS containing 5\% Triton X-100 (PBST). Brains were then agitated for $5 \mathrm{~h}$ in PBST containing 10\% goat serum (PBST-GS) and were transferred to fresh PBST-GS containing rabbit anti-GABA (Sigma) at 1:100 dilution. After incubation for $5 \mathrm{~d}$, brains were washed for $4 \mathrm{~h}$ in PBST at room temperature and transferred to PBST-GS containing Alexa Fluor 633conjugated goat anti-rabbit IgG (Invitrogen) at 1:100 dilution and were incubated for $5 \mathrm{~d}$. They were then washed in PBS, dehydrated through ethanol series, cleared in methyl salicylate, and examined by confocal laser scanning microscopy. Confocal stacks were analyzed using public domain software ImageJ (National Institutes of Health).

Data analysis. All analyses were performed using custom programs in MatLab (MathWorks). Peristimulus time histograms (PSTHs) of neural activity were estimated by convolving instantaneous firing rate (computed in $20 \mathrm{~ms}$ bins, averaged over trials) with a $200 \mathrm{~ms}$ Gaussian function. Responsiveness of a neuron was determined by an automated algorithm: a neuron was considered responsive to a stimulus if the PSTH in a $2 \mathrm{~s}$ period following stimulus onset changed by $>3.5$ SD from background (Perez-Orive et al., 2002); responsiveness determined by the algorithm was consistent with visual inspection. Sliding-window crosscorrelations between the LFP and LHNs were computed using the "xcov" function in MatLab (MathWorks) with the "coeff" normalization option (to minimize the effects of DC shifts and amplitude changes) on $5-40 \mathrm{~Hz}$ bandpass-filtered LFP and unfiltered LHN recordings with $150 \mathrm{~ms}$ windows and $50 \mathrm{~ms}$ shift interval and finally were averaged over multiple trials for a given cell-odor pair. Cross-correlograms between the LFP and the membrane potentials of LHNs (see Fig. 4) were computed using filtered LHN and LFP recordings; LFP autocorrelograms were computed similarly. The strength of spontaneous cross-correlation was estimated by computing the cross-power spectral density between the LFP and the membrane potential of an LHN [both signals centered and scaled with the "zscore" function in MatLab (MathWorks)] in a $2 \mathrm{~s}$ period before odor onset and integrating it over the $15-30 \mathrm{~Hz}$ frequency band. Phases of spikes in LHNs with respect to the concurrent cycle of the LFP were estimated by determining their positions relative to the previous ( 0 radian) and the next ( $2 \pi$ radian) peak in the simultaneously recorded LFP, which was bandpass filtered $(15-30 \mathrm{~Hz})$. Phases of giant GABAergic neuron (GGN) output (which is nonspiking) with respect to the LFP were estimated by recording GGN membrane potential and averaging the rows of the GGN-LFP cross-correlograms (i.e., over time) in a $1 \mathrm{~s}$ response period and determining the positions of the peaks immediately before ( 0 radian $)$ and immediately after $(2 \pi$ radian $)$ the position of zero time lag.

Classification task. Because we could not determine with certainty the total number of neurons in each LHN class, we restricted our analysis to the information content of individual LHNs and report the success rate of an algorithm to classify odors given the responses of each class of LHN. We represented the information content of LHNs with vectors of spikes binned in $50 \mathrm{~ms}$ windows (selected with reference to the strong synchrony of LHNs with the LFP at $\sim 20 \mathrm{~Hz}$ ). For all LHNs, we performed pairwise classification only between an alcohol odor (hex or oct) and a ketone (chx) to ensure accuracy estimates were not biased by the differences in the odors tested for different cells. The classifier first generated a template for each odor by calculating the centroid of the vectors from training trials ( $50 \%$ of all trials) and then classified the remaining (test) trials based on Euclidean distance from the two templates (Stopfer et al., 2003). An accuracy estimate was derived by counting the fraction of correct classifications (expected chance performance is 0.5 ). Another classifier, based on the $k$-means algorithm for unsupervised classification, provided similar results (data not shown).

\section{Results}

\section{LHNs are diverse}

Neurons were impaled, often in a neurite, in the LH region (Fig. 1, top left) with sharp intracellular electrodes, and their responses to a panel of odorants, and in some cases to a light or a sound stimulus, were tested (see Materials and Methods). Recordings were made from a total of 260 cells in 102 animals, providing $\sim 900$ cell-stimulus combinations, with up to 10 trials for each (Table 1). LFPs were recorded simultaneously in the MB calyx using an extracellular electrode to measure odor-evoked oscillations. We were able to fully label approximately one-quarter of the recorded neurons with a fluorescent dye, revealing a surprising diversity of neuron morphology. Although previous studies in Drosophila based on available lines of genetically labeled neurons have reported three or four classes of neurons in the $\mathrm{LH}$ (Tanaka et al., 2004; Jefferis et al., 2007; Yu et al., 2010), we found 10 very distinct morphological classes (Fig. 1; Table 1); examples of each class were observed in at least two brains, and each responded to odors. The cell bodies and primary neurites of neurons within a class were found in consistent locations (Fig. 2A, B, see examples). We refer to all neurons (other than PNs) with neurites in the LH as LHNs to distinguish them from a subset, the LHIs previously described by Perez-Orive et al. (2002). Although PNs send processes to this region, we did not record from them; none of our recordings showed the response characteristics of PNs (phase preference of spiking with respect to the LFP, elaborate and odor-specific spiking patterns), and none of the cells we filled in this region showed the distinctive morphology of a PN. Three of the classes we observed (C1, C5, C6) have been previously reported in the locust. $\mathrm{C} 1$ shows the same morphology 

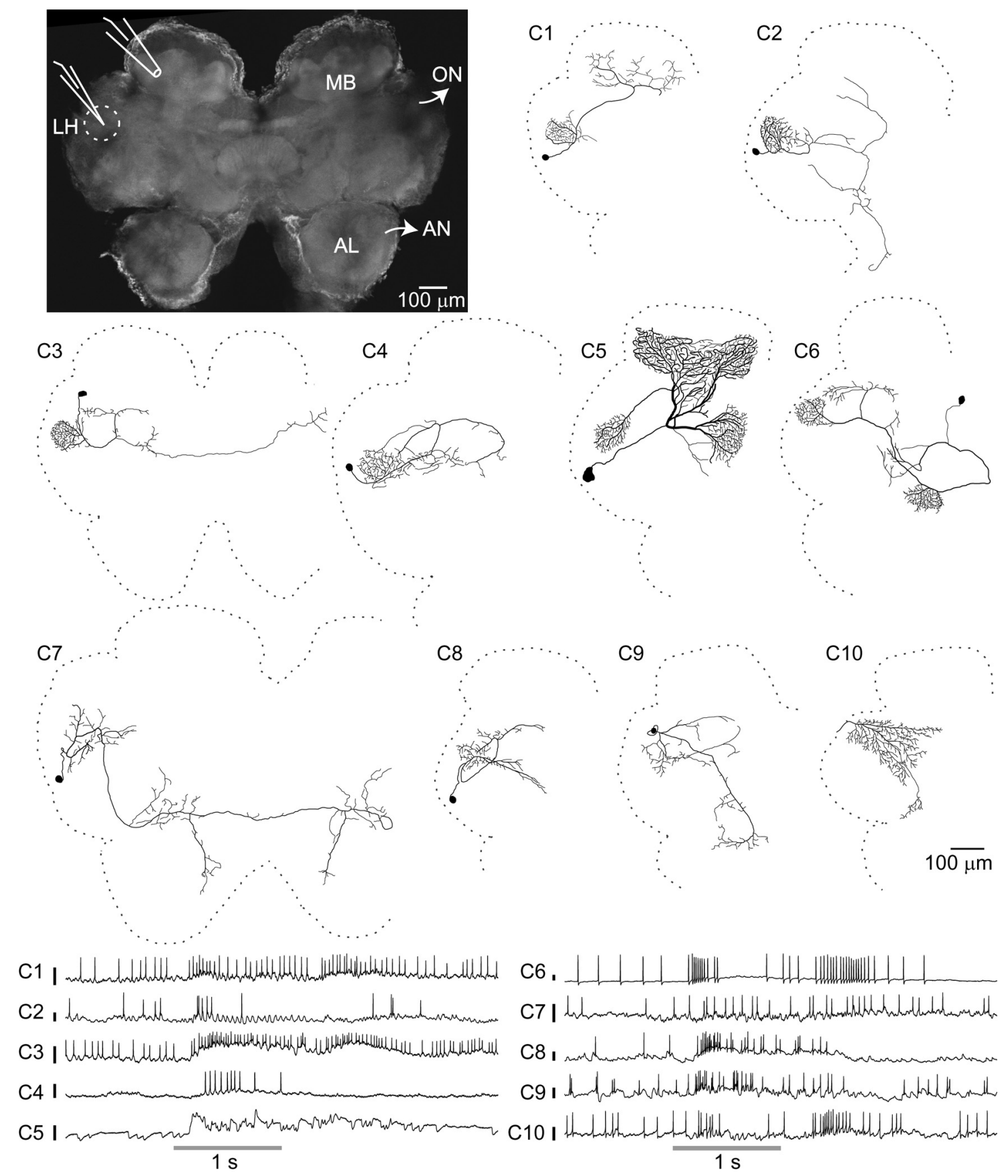

Figure 1. Anatomical and electrophysiological survey of the LH. Top left, Flattened confocal stack of an unlabeled locust brain. Dotted circle, LH. AN, Antennal nerve; ON, optic nerve. Sharp intracellular recordings were made from LHNs, and simultaneous extracellular LFP recordings were made from the ipsilateral MB calyx. Middle, Ten morphologically distinct classes of LHNs, illustrated with hand-traced intracellular dye fills. Somata of C10 neurons were not detected. Bottom, Representative intracellular recordings from the $10 \mathrm{LHN}$ classes, showing their responses to odor presentations (gray bars). Calibration bars, $10 \mathrm{mV}$.

defining the LHIs (Perez-Orive et al., 2002). C5 is the GGN that provides feedback inhibition to the KCs (Papadopoulou et al., 2011). C6 neurons, which also arborize in the $\beta$-lobe of the $\mathrm{MB}$, have been previously reported as one of the anatomical subtypes of $\beta$-lobe neurons ( $\beta$-LNs) belonging to the set of MB output cells (MacLeod et al., 1998; Cassenaer and Laurent, 2007).

In sharp contrast to the sparse spiking observed in KCs, which are targets of the PNs in the MB (Perez-Orive et al., 2002; Stopfer et al., 2003; Ito et al., 2008; Turner et al., 2008; Honegger et al., 2011), LHNs typically fired spontaneously and showed strong responses to odors (Fig. 1, bottom). These responses varied from simple increases or decreases in firing rate to more complex temporal patterns of spikes. Although different classes of LHNs generated somewhat different spiking patterns, responses to different odors within a class were not always more similar to each other than to the responses of other classes. Thus, it was generally not possible, with the sole exception of C5, to use these responses to accurately identify the class of a neuron. We therefore used morphological criteria to classify neurons. C5 neurons (GGN) showed graded odor responses, did not respond with action potentials to any odor or current stimulus, and could be 
Table 1. LHN classes

\begin{tabular}{|c|c|c|c|c|c|c|c|c|c|}
\hline $\begin{array}{l}\text { Class ID } \\
\text { (common name) }\end{array}$ & $\begin{array}{l}\text { Location of cell } \\
\text { body }\end{array}$ & $\begin{array}{l}\mathrm{LH} \\
\text { arborization }\end{array}$ & Other regions of arborization & $\begin{array}{l}\text { Times } \\
\text { filled }\end{array}$ & $\begin{array}{l}\text { Cell stimulus pairs } \\
\text { recorded for filled } \\
\text { neurons }\end{array}$ & $\begin{array}{l}\text { Trials per recording } \\
\text { (mean } \pm \text { SD) }\end{array}$ & $\begin{array}{l}\text { GABA-positive neurons/ } \\
\text { total GABA stains } \\
\text { obtained }\end{array}$ & Comments & References \\
\hline C1 (LHI) & Middle lateral pr & Dense & MB calyx & 7 & 11 & $9.0 \pm 1.6$ & $0 / 2$ & $\begin{array}{l}\text { Previously proposed to mediate feed- } \\
\text { forward inhibition to KCs }\end{array}$ & Perez-Orive et al., 2002 \\
\hline $\mathrm{C} 2$ & Middle lateral pr & Dense & $\begin{array}{l}\text { A branch from LH bifurcates: one part goes to } \\
\text { posterior pr and another to anterior pr } \\
\text { and ventral deutocerebrum }\end{array}$ & 2 & 7 & $9.3 \pm 1.9$ & $a$ & & \\
\hline C3 & Posterior lateral pr & Dense & $\begin{array}{l}\text { Looping projections in middle/posterior pr; } \\
\text { one branch reaches contralateral LH }\end{array}$ & 6 & 25 & $8.2 \pm 2.4$ & $0 / 3$ & Projects bilaterally & \\
\hline$C 4$ & Middle lateral pr & Dense & Looping projections in middle/posterior pr & 3 & 10 & $8.7 \pm 2.4$ & $1 / 1$ & & \\
\hline C5 (GGN) & Middle lateral pr & Dense & $\begin{array}{l}\text { MB } \alpha \text {-lobe (input); MB calyx (output); MB } \\
\text { pedunculus }\end{array}$ & 15 & 111 & $9.9 \pm 0.6$ & $3 / 3$ & $\begin{array}{l}\text { Nonspiking responses; nine unfilled } \\
\text { neurons could also be identified } \\
\text { as GGNs from their characteristic } \\
\text { responses }\end{array}$ & $\begin{array}{l}\text { Liu and Davis, 2009; } \\
\text { Papadopoulou et al., } \\
2011\end{array}$ \\
\hline $\mathrm{C} 6(\beta-\mathrm{LN})$ & Posterior medial pr & Dense & $\begin{array}{l}\text { MB } \beta \text {-lobe (input); MB pedunculus; looping } \\
\text { projections in posterior lateral pr }\end{array}$ & 9 & 46 & $8.6 \pm 3.0$ & $0 / 3$ & $\begin{array}{l}\text { One of the two types of output } \\
\text { neurons reported in MB } \beta \text {-lobe }\end{array}$ & $\begin{array}{l}\text { MacLeod et al., 1998; } \\
\text { Cassenaer and Laurent, } \\
\text { 2007; Papadopoulou et al., } \\
2011\end{array}$ \\
\hline$C 7$ & Anterior lateral pr & Sparse & $\begin{array}{l}\text { Ipsilateral and contralateral anterior medial } \\
\text { pr: one branch goes toward ventral } \\
\text { deutocerebrum }\end{array}$ & 4 & 15 & $9.4 \pm 1.7$ & $0 / 2$ & Projects bilaterally & \\
\hline C8 & Middle lateral pr & Sparse & Posterior lateral pr, anterior medial pr & 12 & 47 & $8.7 \pm 2.1$ & $2 / 2$ & & \\
\hline C9 & Posterior lateral pr & Sparse & $\begin{array}{l}\text { Posterior lateral pr, anterior medial pr, } \\
\text { ventral deutocerebrum }\end{array}$ & 2 & 15 & $7.9 \pm 2.5$ & $a$ & & \\
\hline C10 & Not identified & Sparse & $\begin{array}{l}\text { Posterior and middle lateral pr: one branch } \\
\text { goes toward anterior medial pr }\end{array}$ & 2 & 13 & $8.1 \pm 2.1$ & $a$ & $\begin{array}{l}\text { Multimodal responses; also shown by } \\
10 \text { unfilled neurons }\end{array}$ & \\
\hline
\end{tabular}

Descriptions of 10 morphological classes of LHNs identified from intracellular fills. Because the boundaries of the LH are not clearly visible in individual fills, we could only determine whether a neuron had dense or sparse projections in the region. pr, Protocerebrum.

${ }^{a}$ Classes for which GABA stains were not available.

unambiguously identified by their characteristic subthreshold activity patterns (Papadopoulou et al., 2011).

\section{LHIs do not provide feedforward inhibition to the KCs}

Perez-Orive et al. (2002) suggested one class of LHN, the LHIs, provides feedforward inhibition to the KCs in the MB calyx. We recorded from seven neurons (class $\mathrm{C} 1$ ) that match the key anatomical characteristics of LHIs: they have somata in a cluster near the $\mathrm{LH}$, form dense arborizations in the $\mathrm{LH}$, and project to the calyx of the MB. Consistent with the previous description (PerezOrive et al., 2002), we found these neurons responded strongly and reliably to all tested odors with spikes and subthreshold membrane potential oscillations (Fig. 1).

We obtained clear immunostaining against GABA in two brains containing LHIs, with responses characterized by intracellular recordings and morphologies identified by intracellular dye injection. Both revealed the same surprising result: neither LHI labeled positively for GABA. In both brains, other neurons clustered near the LHI were strongly labeled (Fig. 2A,B), indicating our stains were successful and reproducible. These results suggested that, although the LHIs are located near GABAergic cells, the LHIs themselves are not GABAergic. We re-examined the evidence presented by Perez-Orive et al. (2002). These authors did not show immunostaining for individual LHIs, but rather described two types of results from anti-GABA screening of the whole brain. First, they found a cluster of $\sim 60$ GABAergic cells near the cell bodies of the LHIs. Second, they identified a GABAergic tract between the LH and the MB calyx, potentially bearing the axons of the LHIs. They also noted that dendrites of KCs intermingle with GABAergic fibers in the calyx region of the MB (Leitch and Laurent, 1996) and that KCs show odorelicited IPSPs.

Our results suggest other types of neurons underlie all these observations. Class C8 neurons, which have cell bodies near those of the LHIs but do not project to the calyx (Fig. 1), labeled posi- tively for GABA (Fig. 2C). Moreover, C8 cells were identified more frequently (12 times) than the LHIs (seven times), suggesting $\mathrm{C} 8$ cells constitute at least a portion of the cluster thought to contain the LHIs. We also identified the GABAergic tract (Fig. $2 \mathrm{D}$ ) between the $\mathrm{LH}$ and the MB calyx described by Perez-Orive et al. (2002). However, our intracellular fill of GGN (Fig. 1, class C5) followed by immunostaining for GABA revealed this tract is actually a branch of GGN (Fig. 2D). Moreover, nearly all the GABAergic fibers we repeatedly observed in the MB calyx overlapped precisely with arbors of GGN, indicating GGN provides by far the greatest source of inhibitory input to the KCs (Fig. 2 E). We identified additional GABAergic fibers of unknown origin running along the pedunculus to the accessory calyx (but not projecting to the $\mathrm{LH}$ ); because KCs in the olfactory system do not project to the accessory calyx (Laurent and Naraghi, 1994), these fibers are unlikely to inhibit the KCs. Thus, of the two types of neurons (GGN and LHIs) proposed to inhibit KCs, only GGN appears to perform this role.

\section{GGN can account for most roles attributed to LHIs}

The LHIs described by Perez-Orive et al. (2002) were suggested to play two important roles in odor coding.

Piecewise decoding of PN input by the KCs

The spikes of the LHIs, biased by their inputs from rhythmically driven PNs to occur in a specific phase position in every oscillation cycle, have been proposed to provide a mechanism for KCs to decode input from PNs cycle by cycle. This helps KCs to function as coincidence detectors (Perez-Orive et al., 2002, 2004) responding only to synchronized firing from multiple presynaptic PNs (Jortner et al., 2007). It is unclear whether the graded output of the nonspiking GGN could perform a similar role. It was shown recently that GGN output intensity correlates with the overall magnitude of the LFP oscillations when viewed across cycles (Papadopoulou et al., 2011). Does this output vary with 


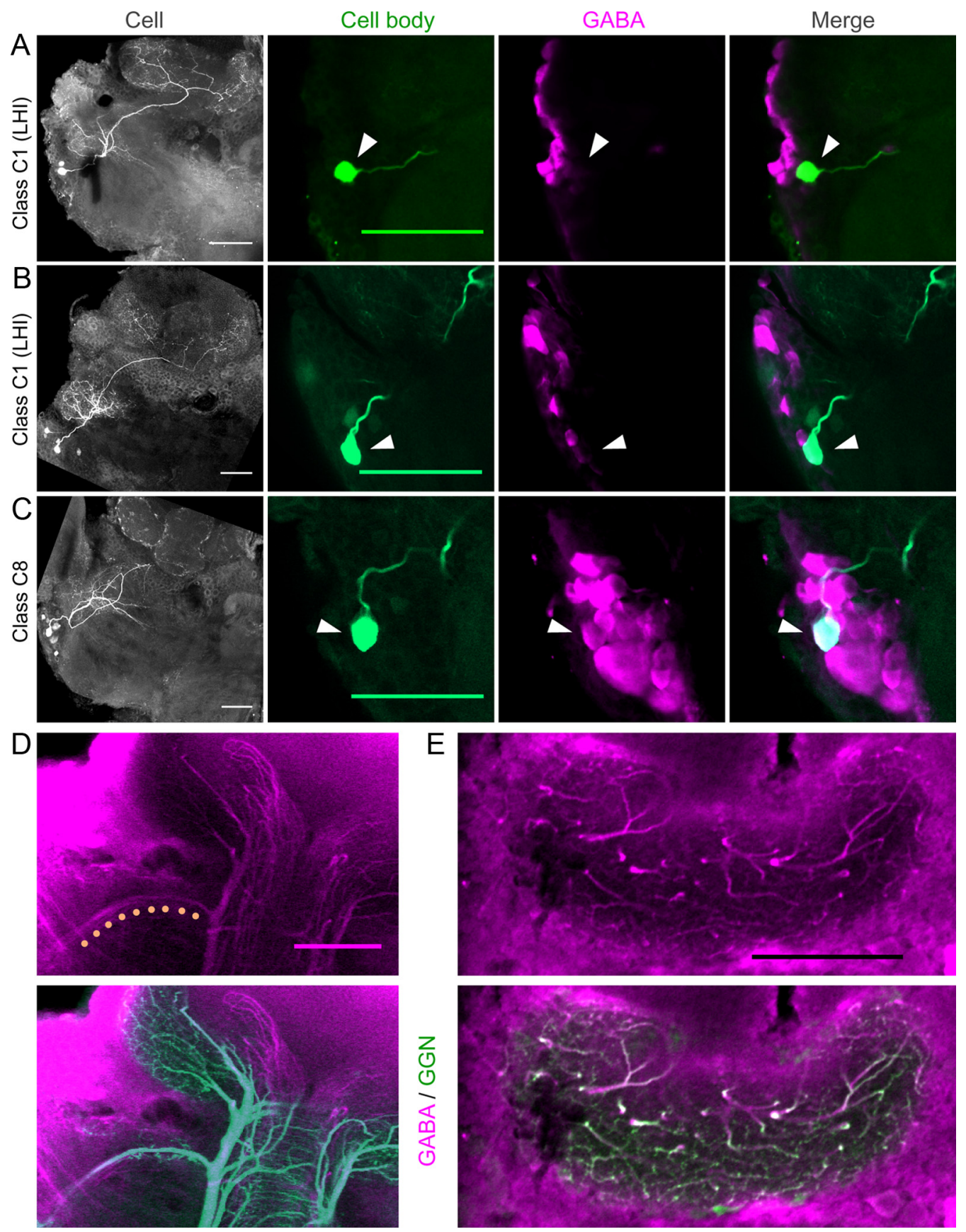

Figure 2. Source of GABAergic inhibition to the MB calyx. A-C, Dye-filled LHls projecting to the MB calyx do not label positively for GABA. $A, B$, LHN class $C 1$ LHls. Left, Flattened confocal stack shows neuron's morphology (showing left half of the brain; compare with Fig. 1 for orientation). Middle to right, Overlap between the intracellular fill (green) and the GABA immunostain (magenta) in a region near the cell body (arrowheads). LHls are GABA negative but are located near GABA-positive cells. C, LHN class C8. This LHN is GABA positive but does not project to the MB calyx. D, Top, GABA immunostain shows a GABAergic tract between $\mathrm{LH}$ and MB calyx (dots). Bottom, Intracellular fill of GGN in the same brain shows this tract is a branch of GGN. $E$, Top, GABA immunostain shows GABAergic fibers in the MB calyx. Bottom, Intracellular fill of GGN in the same brain shows most or all of these fibers are branches of GGN. Scale bars, $100 \mu \mathrm{m}$.

phase within a cycle? Analyzing the within-cycle phase relationship between GGN and the simultaneously recorded LFP, we made the following observations. First, on presentation of odors, GGN membrane potential showed strong oscillatory synchrony with the LFP (Fig. 3A). Second, the phase difference between GGN membrane potential and the LFP was usually between $180^{\circ}$ and $210^{\circ}$ (Fig. 3 B1,B2); that is, the peaks of GGN output occurred near the troughs of the LFP. Third, the phase remained unchanged for different odors (Fig. 3C). Together, these observations show the inhibitory drive to the KCs from GGN is strongest at a specific phase of the oscillatory cycle, and this phase remains stable across different odors, potentially enforcing uniformly sized integration windows within each oscillation cycle over which KCs could integrate the PN input (Fig. 3D).

Maintaining sparseness of spiking in KCs across odor concentrations

Increases in odor concentration increase the coincidence of firing among PNs, but, puzzlingly, do not alter the sparseness of firing in the coincidence detecting KCs (Stopfer et al., 2003). A computational model based on feedforward inhibition from the LHIs to the KCs suggested that concentration-driven changes in the 
phase of spiking of LHIs could adjust the integration windows for KCs (Assisi et al., 2007), thus maintaining the sparseness of response in KCs elicited by different concentrations of odors. However, we found the phase of GGN output did not vary with odor concentration (Fig. 3E). Could GGN maintain the sparseness of firing in KCs by another mechanism? Recently, a computational model (Papadopoulou et al., 2011) suggested that GGN can help maintain the sparseness by increasing its net inhibitory output with the odor concentration and supported this prediction with tests using one odorant. We confirmed and modestly extended these results by comparing GGN output elicited by low and high concentrations of three different odorants. We found increasing the concentration of each odor significantly increased the amplitude of GGN output (Fig. $3 F$ ), supporting the role of GGN in maintaining sparse firing of KCs but by a mechanism different from that proposed for the LHIs.

\section{LHNs and innate odor preferences}

Next, we asked whether the connectivity and response properties of different classes of LHNs are consistent with a labeled line model of encoding innate preferences for odors (Tanaka et al., 2004; Ruta et al., 2010). LHNs are commonly thought to have specific connectivity to PNs and very selective responses to odors (Luo et al., 2010). Our intracellular fills from LHNs showed that classes C1, C2, $\mathrm{C} 3$, and $\mathrm{C} 4$ form dense, ball-like projections exclusively in the LH (Fig. 1), suggesting these neurons receive densely convergent input from large numbers of PNs. Such input would be expected to generate, even in the absence of odors, membrane-potential fluctuations that closely resemble the LFP, which is established by converging input from many PNs in the calyx (Laurent and Naraghi, 1994). Indeed, we found the membrane potentials of LHN classes C1-C4 were tightly synchronized with the LFP even in the absence of odor (Fig. 4A1); these measures resembled an autocorrelation of the LFP (Fig. 4A2). Classes C1-C4 showed strong spontaneous synchrony with the LFP before odor onset, significantly more than did other classes (Fig. 4A4). These anatomical and physiological results both suggest classes C1, C2, C3, and C4 LHNs receive massively convergent input from $\mathrm{PNs}$, consistent with our observation that these cells responded to all the odors we tested.

Although classes C5 (GGN) and C6 ( $\beta$-LN), too, formed dense projections in the LH, they also arborized heavily in the $\alpha$-lobes and the $\beta$-lobes of the MB, respectively, and are known to receive their primary input from these lobe areas (Cassenaer and Laurent, 2007; Papadopoulou et al., 2011). Further, it was re-
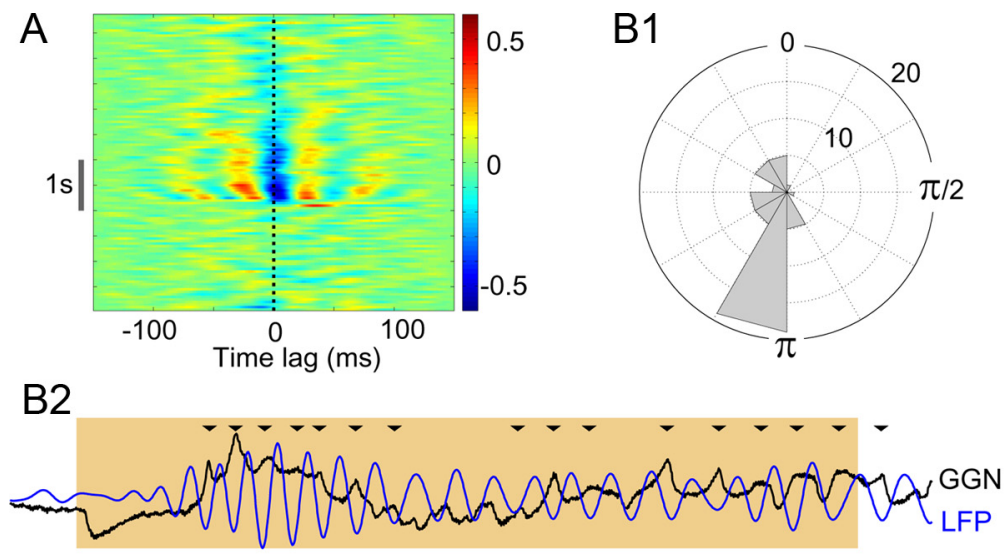

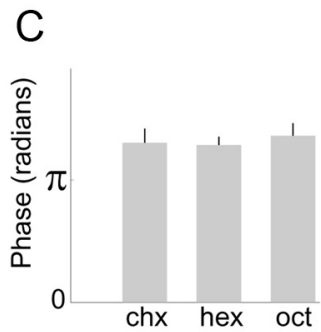

$\mathrm{D}$
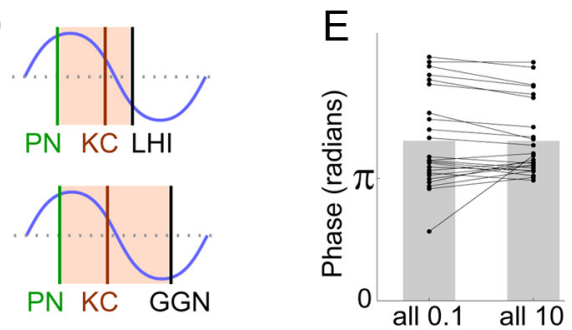

$\mathrm{F}$
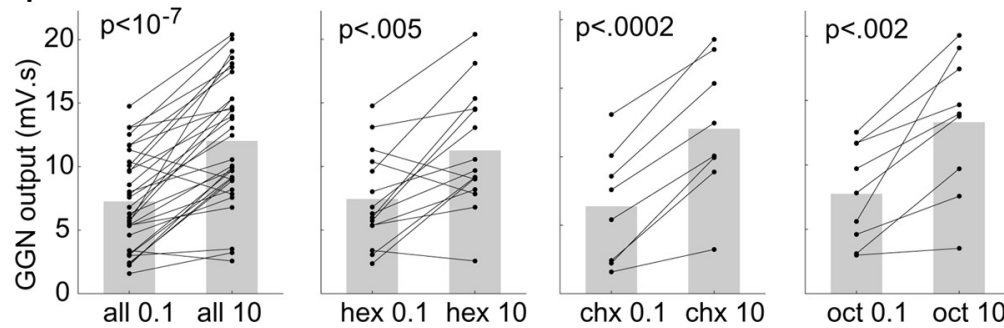

Figure 3. GGN-mediated feedback inhibition is consistent with the regulation of temporal integration in KCs. $\boldsymbol{A}$, Sliding window cross-correlation diagram (average of 10 trials) shows strong phase locking between GGN and the LFP; red and blue bands indicate, ( of GGN and the LFP (filtered 15-30 Hz) during an odor presentation (colored background). In most oscillation cycles, peak GGN output occurs near the trough of the LFP (arrowheads). C, Average GGN phase for three different odors ( $n=10,24$, and 14 comparing the integration windows for two models. Top, Presumed inhibitory spikes in LHIs, occurring after the excitatory spikes in PNs, were proposed to create brief integration windows (colored background) for the KCs within each oscillatory cycle. Bottom

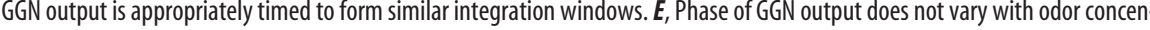
: pairwise comparison of GGN phase evoked by $0.1 \%$ and $10 \%$ odor concentrations; $n=24$ cell- odor combinations from 12

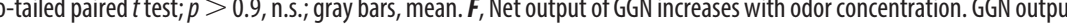
(membrane potential above the baseline integrated for $2 \mathrm{~s}$ after odor onset) evoked by $0.1 \%$ and $10 \%$ concentrations of odor (all combined in the first panel); $n=15,8$, and 9 cell-odor pairs, respectively, for hex, chx, and oct; two-tailed paired $t$ tests.

cently shown that silencing KCs by depolarizing GGN also silenced $\beta$-LNs (Papadopoulou et al., 2011), indicating $\beta$-LNs do not receive direct input from PNs. The responses of GGN and $\beta$-LNs were different from those of LHN classes C1-C4; the former synchronize with the LFP only during odor presentations (Figs. 3A, 4A3,A4). Finally, consistent with results suggesting only classes $\mathrm{C} 1-\mathrm{C} 4$ receive direct and converging input from $\mathrm{PNs}$ (see next section), we found concentration-dependent changes in the phases of the spikes of classes $\mathrm{C} 1-\mathrm{C} 4$ but not in those of $\beta$-LNs or in the phases of GGN graded output (Fig. 3E). LHN classes C7-C10 did not show spontaneous cross-correlations with the LFP (Fig. 4A4) and did not appear to receive massively convergent $\mathrm{PN}$ input. 

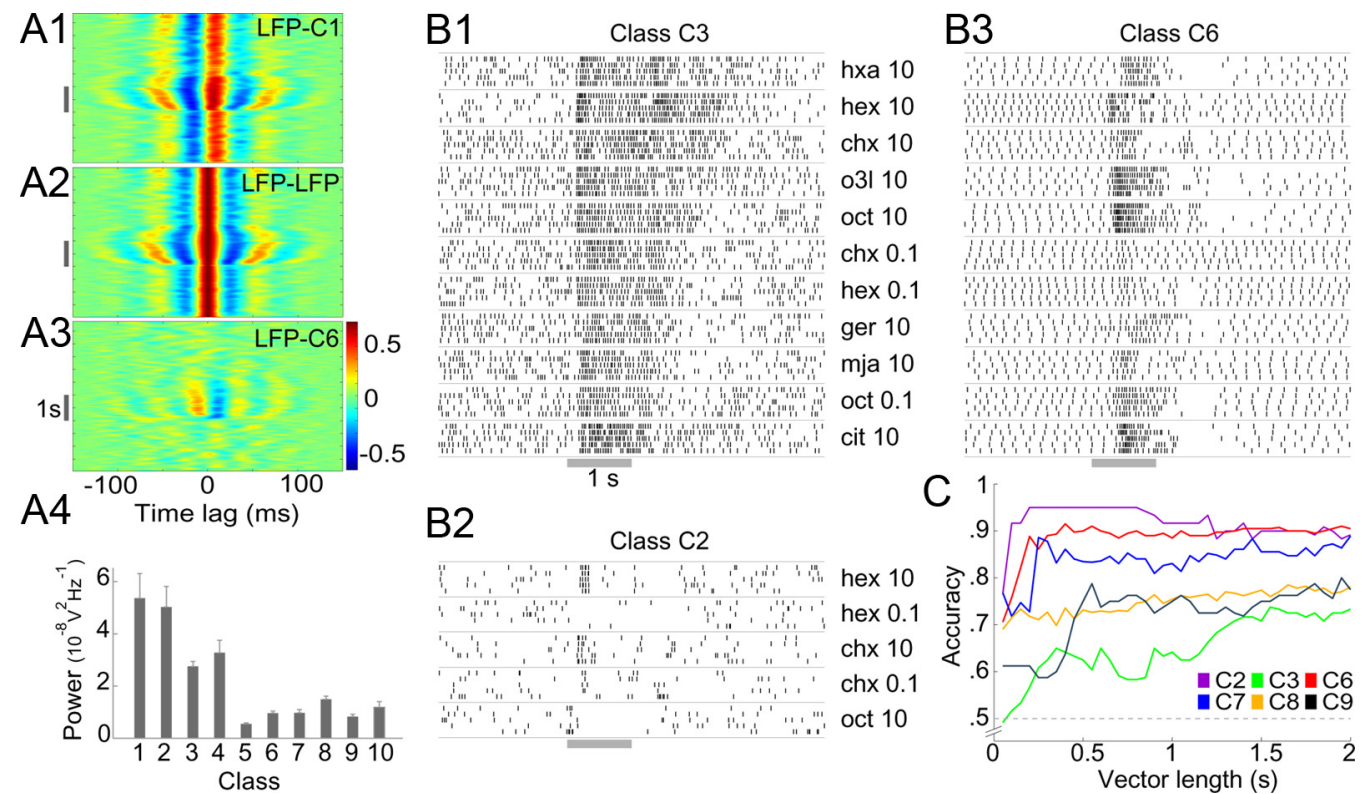

Figure 4. Response characteristics of LHNs. $A$, Different types of LHNs vary in the extent to which their activity is correlated with the LFP. $\boldsymbol{A} 1$, Cross-correlogram of subthreshold membrane potential activity in a class $\mathrm{C} 1 \mathrm{LHN}$ with the LFP, $\boldsymbol{A 2}$, LFP autocorrelogram for comparison. $\boldsymbol{A}$ 3, Cross-correlation of a $\beta$-LN (class (6) and the LFP. Gray bar, 0 dor pulse. Note strong correlations in $\boldsymbol{A} \mathbf{1}$ and $\boldsymbol{A} \mathbf{2}$ even in the absence of odor. $\boldsymbol{A 4}$, Cross-power spectra (see Materials and Methods; mean \pm SEM for all recordings in a class), measured over a 2 s period before odor onset, show classes $(1-C 4$ have stronger spontaneous synchrony with the LFP than remaining classes $C 5-C 10(p<0.0004$; two-tailed, two-sample $t$ test; $\mathrm{df}=8)$. $\boldsymbol{B}$, Responses of different LHNs contain varying amounts of information about odor identity. Raster plots illustrate the responses of one representative neuron each from classes $C 2$ (B2), 3 ( $(B 1)$, and C6 (B3), to multiple odors (five trials each, 10 s interstimulus interval). Gray bar, odor pulse (for odor list, see Materials and Methods). C, Pairwise odor classification accuracy, as a function of the sample duration (vector length) for different classes of $L H N$ s (chance level, 0.5$) . n=3,6,10,6,13$, and 4 odor pairs for classes $\mathrm{C}_{2}, \mathrm{C}, \mathrm{C}, \mathrm{C}, \mathrm{C}, \mathrm{C}$, and $\mathrm{C}$, respectively. Classes with fewer than three odor pairs or nonspiking responses (C5) were not analyzed.

GGN has been shown to respond to all tested odors (Papadopoulou et al., 2011); our recordings confirmed this (data not shown). Here, we report the selectivity of other classes of LHNs. In total, our dataset included 171 cell-odor pairs from morphologically identified neurons from classes C1-C10 (excluding GGN). Of these, 170 pairs cleared an automated algorithm threshold for detecting a significant response to odor presentation (see Materials and Methods); visual inspection yielded nearly identical results and showed that the single cell-odor pair whose response did not clear the algorithm threshold contained a small but reliable inhibitory component.

Thus, all the LHNs we identified (classes C1-C10) responded to all tested odors; notably, in this respect LHNs are less selective than PNs (Wehr and Laurent, 1996). Further, many LHNs showed anatomical and physiological evidence of receiving densely convergent input from PNs, contradicting an earlier proposal (Tanaka et al., 2004). The odorant panel we used in this study, though limited in size, included odors with different types of ecological relevance. Grass volatiles hexanol and hexanal included in the panel are innately attractive to locusts (Sun et al., unpublished observations); yet, responses of LHNs to these odorants were not distinct from responses to other odorants. Because C1-C10 LHNs responded broadly to our panel of odorants, we can infer that these LHNs are not specialized to respond exclusively to any potentially relevant odors that we did not test. Individual LHNs could show excitatory or inhibitory responses to multiple odors (Fig. $4 B 1, B 2$ ) or both excitation and inhibition in sequence in response to a single odor (Fig. 4B3). The odorevoked firing patterns of some of these broadly responsive neurons, like those of PNs, contained information that could be used to classify odor identity (Fig. 4B,C; see Materials and Methods). After 300-500 ms of odor onset, a time period found sufficient for making behavioral decisions (Vickers and Baker, 1996), the classification success rate of different classes of LHNs varied widely, from $60 \%$ to $95 \%$ (Fig. 4C), consistent with the functional diversity of cell types in the LH. Together, our results lend no support to the idea that LHNs, in general, act as labeled lines for mediating highly selective behavioral responses to specific, innately meaningful odors.

\section{Simple coding of stimulus intensity}

What other functions might the LH serve? Because LHNs are fewer in number than and receive convergent input from PNs, we considered that LHNs might serve, in part, to extract general features of odorants. A computational model has suggested converging input from PNs could cause spikes in LHNs to occur with a phase preference that varied with, and thus contained information about, odor concentration (Assisi et al., 2007). To test this idea experimentally, we measured the phases of spiking relative to the LFP in LHNs that appear to receive convergent input from PNs (classes C1-C4).

As predicted by the computational model, we found that spike phase indeed advanced in these neurons; that is, spikes arrived earlier in each oscillation cycle as the odor concentration increased (Fig. 5A1,A2). These changes, though small in magnitude, were significant and reliable across different cell-odor pairs (Fig. 5A3). In comparison, $\beta$-LNs (class C6), which did not appear to receive convergent input from PNs, did not show such phase specificity across odor concentrations (Fig. 5B). Further, we found significantly more spikes were elicited by higher concentrations of odors in LHN classes C1-C4 (Fig. 5C,D). Thus, unlike their presynaptic partners, the LHNs receiving convergent $\mathrm{PN}$ input appeared to encode stimulus intensity in their net firing rates and in the phases of their spikes.

\section{Bilateral integration}

The olfactory systems of many animals, including humans (but excluding Drosophila; Stocker et al., 1990), consist of two lateral- 


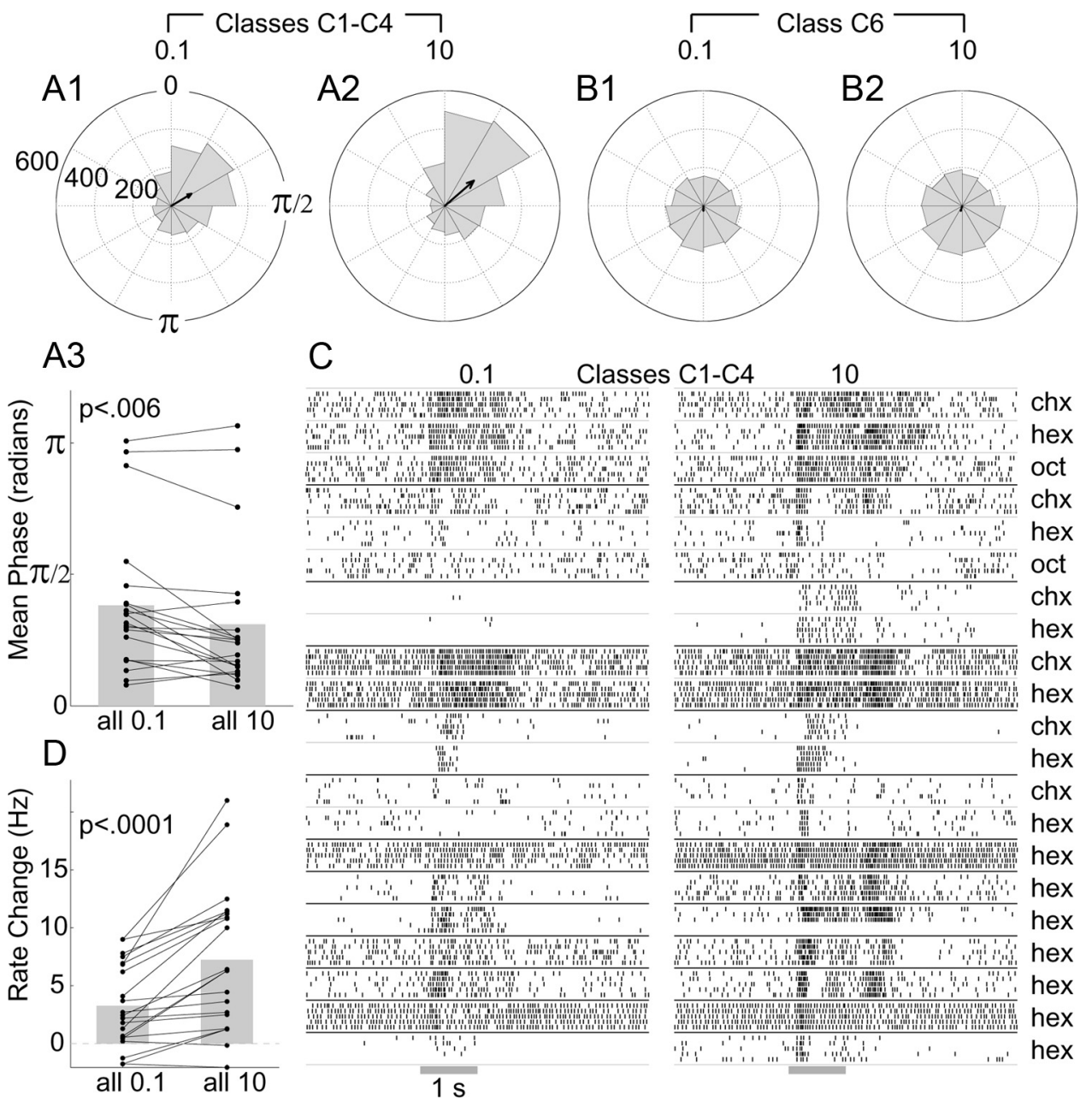

Figure 5. Coding odor concentration in C1-C4 LHNs. A, Spike phase positions in C1-C4 LHNs advance with odor concentration, shown by polar histograms of responses to $0.1 \%$ (A 1) and 10\% (A2) odor concentrations ( $>2200$ spikes for each concentration). Arrows show vector average. Note the decrease in phase evoked by the higher concentration. $A 3$, Pairwise comparison of the mean phase for $(1-C 4$ recordings evoked by low and high concentrations (two-tailed paired $t$ test; $n=21$ cell-odor combinations, 13 cells; see $C$ ) shows significant differences. $\boldsymbol{B}$, Phase positions of spikes in $\beta$-LNs (class (6, >2200 spikes) (B1, B2) show little phase preference; vector average is close to zero. C, Raster plots show responses of the 13 C1-C4 LHNs to both $0.1 \%$ and $10 \%$ concentrations of various odors. $\boldsymbol{D}$, Pairwise comparison of the net change in firing rate evoked by high and low concentrations (average firing rate in a 2 s period from odor onset minus the average background firing rate); two-tailed paired $t$ test for the same $(n=21)$ cell-odor combinations.

ized, separate pathways in the initial stages of processing. In the locust, for example, none of the well studied olfactory neurons, including the olfactory receptor neurons, the local neurons (in the $\mathrm{AL}$ ), PNs, KCs, $\beta$-LNs, and GGN, cross the midline to innervate neurons on the opposite side of the brain. However, behavioral observations in insects (Martin, 1965; Louis et al., 2008) and in mammals (Rajan et al., 2006, Porter et al., 2007) show bilateral integration of olfactory information helps in tracking odors. Which neurons in the olfactory system could mediate this process?

We found that two classes of odor-responsive LHNs in the locust, $\mathrm{C} 3$ and $\mathrm{C} 7$, extend bilateral projections (Figs. 1, 6A), suggesting the $\mathrm{LH}$ may be the first region in the olfactory hierarchy to mediate bilateral integration. Interestingly, C3 neurons project to the contralateral LH. Spikes recorded in C3 and C7 LHNs showed odor-evoked synchrony (Fig. 6C) and phase locking with the LFP (Fig. 6D).

\section{Multimodal integration}

We asked whether the LH might also mediate the integration of olfactory information with other sensory modalities. GGN, despite sending widely sweeping processes throughout the calyx, pedunculus, and the $\alpha$-lobe of the MB (Figs. 1, 2D,E), did not respond to light or sound in any of our recordings (data not shown). However, 12 other neurons in our sample responded to both odors and light (for four examples, see Fig. 6E); their responses were validated with the automated response detection algorithm (see Materials and Methods). These multimodal responses contained varied patterns of excitation and inhibition (Fig. 6E). Two of these neurons could be morphologically identified (class C10) by intracellular fills (Fig. 6B). They showed sparse projections in the ball-like region of $\mathrm{LH}$ associated with PN output (compare with classes C1-C4 in Fig. 1), suggesting these multimodal cells may receive their olfactory input from higher-order cells in the region, possibly other LHNs. None of the multimodal cells we identified showed synchrony with the LFP (Figs. 4A4, 6C).

\section{Discussion}

Compared with the well studied roles of the MB in olfactory coding (Laurent, 2002; Masse et al., 2009) and learning (Keene and Waddell, 2007; Davis, 2011), the role of the LH in general olfaction remains unclear. We surveyed the LH with electrophysiological and anatomical techniques to evaluate hypotheses about functions this area has 


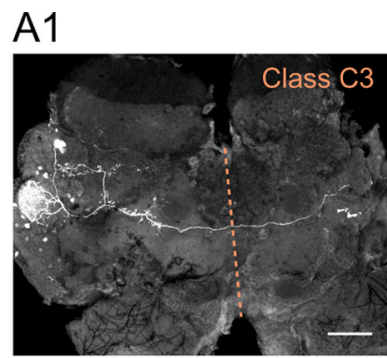

\section{A2}
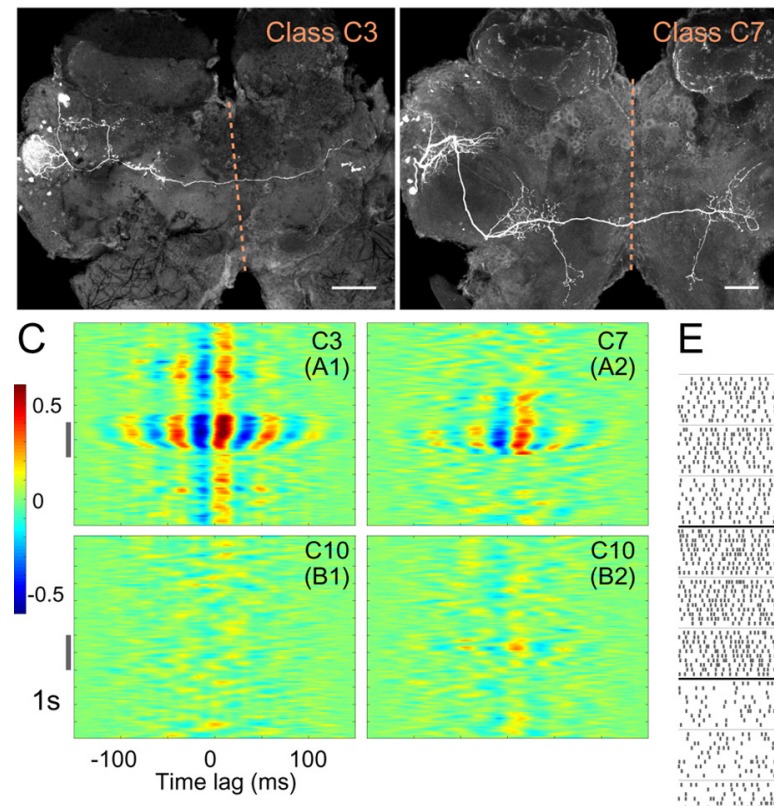

D

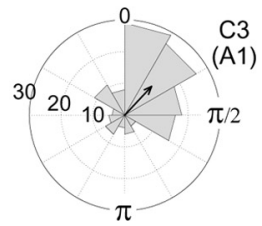

$\mathrm{C} 7$
(A2)
B1

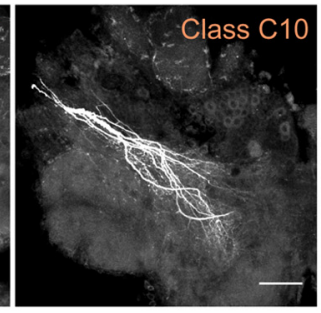

B2
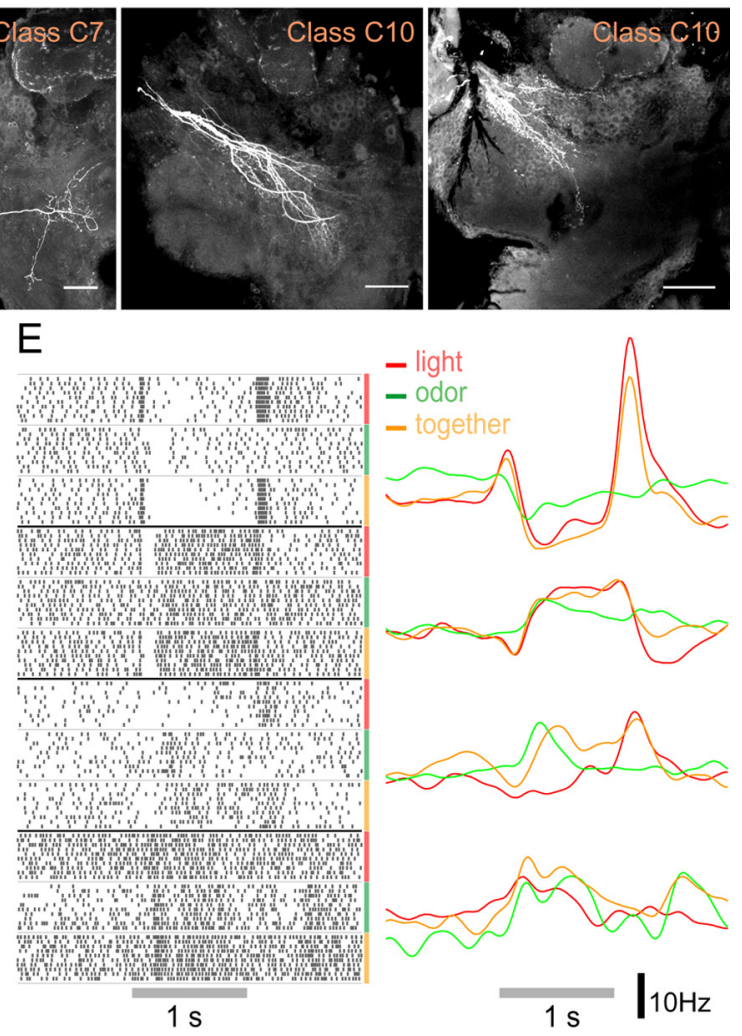

Figure 6. Bilateral and multimodal LHNs. $\boldsymbol{A}$, Intracellular fills of a C3 (A1) and a C7 (A2) LHN, showing bilateral projections. Dotted line, Midline. Scale bars, $100 \mu \mu$ m. $\boldsymbol{B}$, Intracellular fills of two C10 (B1, B2) multimodal neurons. Cell bodies were not detected. C, Cross-correlograms show that responses of C 3 and C7 LHNs correlate well with the LFP but that those of C10 neurons do not. $\boldsymbol{D}$, Polar histograms show phase-preference of spikes in the $\mathrm{C}$ and $\mathrm{C}$ neurons shown in $\boldsymbol{A}$. $\boldsymbol{E}$, Responses of four multimodal neurons to a light stimulus, an odor stimulus, or both stimuli presented together, shown as raster plots (left) and PSTHs (right). All responses shown exceeded the automated response detection algorithm significance threshold (see Materials and Methods).

been proposed to serve. Our dye-fills of individual LHNs in the locust revealed a surprising diversity of neurons, consisting of at least 10 distinct morphological classes (Fig. 1; Table 1).

A class of LHNs, the LHIs, was previously proposed to provide feedforward inhibition to regulate the firing of KCs based on two sets of observations: intracellular dye-fills showing these LHIs send processes to the MB calyx and GABA immunostains showing a cluster of $\sim 60$ GABAergic neurons in the area of LHI somata, and a GABAergic fiber tract between the $\mathrm{LH}$ and the MB calyx (Perez-Orive et al., 2002). Whether the dye-filled LHIs labeled positively for GABA was not tested.

With dye-fill experiments, we identified LHNs matching morphological and physiological descriptions of the LHIs (C1). However, following the same immunostaining technique used by the earlier authors, we found these dye-filled neurons were not GABAergic (although we observed strongly stained GABAergic neurons adjacent to them; Fig. $2 A, B)$. Using this technique we also identified the cluster of GABAergic neurons reported earlier. However, our intracellular fills showed this cluster contained GABA-positive C8 neurons that do not project to the calyx (Fig. $2 C)$. Recent work shows $\beta$-LNs with somata near those of the LHIs (Cassenaer and Laurent, 2007) are inhibitory (Cassenaer and Laurent, 2012), suggesting the GABAergic cluster may also contain these $\beta$-LNs. We found that the GABAergic tract between the $\mathrm{LH}$ and the MB, thought by the earlier authors to carry feedforward inhibition from LHIs, is actually a branch of GGN (Fig. $2 D)$. Moreover, we found that most, and perhaps all, of the GABAergic fibers in the calyx appear to be branches of GGN (Fig. $2 E)$. It remains unknown what functions are served by the $\mathrm{LH}$ branch of GGN. Although a blind-stick sampling procedure cannot definitively rule out the existence of any given neuron and unknown neurons or neurotransmitters may provide additional inhibitory pathways, neither published observations nor our new results provide evidence that GABAergic LHIs exist or that any LHNs provide feedforward inhibition to the KCs.

Our results are consistent with anatomical findings from other insect species. In Drosophila, the anterior paired lateral neuron (APL), which may contribute to olfactory memory, is the only known GABAergic neuron projecting to the calyx (Liu and Davis, 2009; Pitman et al., 2011; Wu et al., 2011); it is morphologically and physiologically similar to the locust GGN (Papadopoulou et al., 2011), although its influence on KCs remains to be tested. In cockroach (Yamazaki et al., 1998) and honeybee (Bicker et al., 1985), only large GABAergic neurons, with GGN-like connectivity, appear to provide substantial inhibitory input to the calyx. We identified in the moth Manduca sexta a single cluster of GABAergic cells that arborizes in the calyx and the lobe areas of the MB (data not shown; Homberg et al., 1987).

Periodic phase-locked inhibition from LHIs was proposed to help the KCs function as coincidence detectors (Perez-Orive et al., 2002). This idea has also influenced thinking about the role of inhibition in the mammalian cortex (Larimer and Strowbridge, 2008; Poo and Isaacson, 2009; Stokes and Isaacson, 2010). We found that the graded output of GGN is tightly phase-locked to the olfactory system's oscillatory cycle with a phase-lag suitable for defining an input integration window in KCs within each cycle (Fig. $3 A-C$ ). Thus, although GGN differs from the presumed LHIs in population size (1 vs 60), output properties 
(graded vs spiking), and mechanism of inhibition (feedback vs feedforward), it may also promote coincidence detection in the KCs by imposing brief, stable integration windows in each oscillatory cycle (Fig. $3 D$ ). Our results are consistent with the finding of Perez-Orive et al. (2002) that infusing a GABA receptor blocker to the $\mathrm{MB}$ reduces the specificity of olfactory responses in the KCs; our results suggest the source of GABA is GGN rather than the LHIs. Other factors are also thought to contribute to the sparseness of KCs, including their high firing thresholds and specialized nonlinear membrane conductances (Perez-Orive et al., 2002, 2004; Turner et al., 2008; Demmer and Kloppenburg, 2009). A theoretical model suggested that adaptive regulation of the strength of the PN-KC synapse could also help maintain this sparseness (Finelli et al., 2008).

Increasing concentrations of odors elicit increasingly coincident spiking across PNs, yet the odor-elicited responses of KCs remain sparse across a broad range of odor concentrations (Stopfer et al., 2003). A computational model suggested GABAergic LHIs could help maintain this sparseness by advancing the spiking phase of feedforward inhibition they provide to KCs as the odor concentration increases (Assisi et al., 2007). Could GGN instead play this role? We found the output phase of GGN remains invariant with concentration, ruling out this mechanism (Fig. 3E). However, GGN appears able to offset increasingly coincident input from PNs in a different way: it counters increasing firing in KCs by feeding back to them stronger inhibition (Fig. 3F) (Papadopoulou et al., 2011). Because strong IPSPs rise faster than weak ones, rhythmic inhibition from GGN could regulate the duration of integration windows.

Neurons of the LH have been proposed to mediate specific responses to odorants that are innately meaningful to the animal (Heimbeck et al., 2001; Jefferis et al., 2007). Some LHNs in Drosophila express sex-specific transcripts of the fruitless gene implicated in courtship behaviors (Yu et al., 2010). Further, recent physiological work identified a cluster of neurons in the LH that respond only to the pheromone 11-cis-vaccenyl acetate (Datta et al., 2008; Ruta et al., 2010). These results suggest pheromones, and possibly other odors involved in sexual behaviors (Grosjean et al., 2011), may be processed by specifically tuned subpopulations of LHNs (Jefferis et al., 2007; Touhara and Vosshall, 2009; Yamagata and Mizunami, 2010). Whether the LH also provides specialized pathways for specific odors processed by the general (nonpheromonal) olfactory system has been unclear. In Drosophila, some anatomical evidence suggests stereotypy and clustering in the projections of genetically labeled PNs in the LH (Tanaka et al., 2004; Jefferis et al., 2007; Lin et al., 2007; Kazama and Wilson, 2009), but whether dendrites of LHNs are restricted to these clusters remains controversial (Tanaka et al., 2004; Jefferis et al., 2007). Nevertheless, the LH, in general, is thought to mediate very specific responses to odors (Luo et al., 2010). Surprisingly, we found in locust that all LHNs we tested (classes C1-C10) responded to all odors. Our anatomical and electrophysiological results show dense convergence of PNs onto LHNs (Fig. 4), providing no support for the idea that LHNs, in general, contribute to a labeled line-like coding of innate preferences for specific odors in the general olfactory system. It remains possible the $\mathrm{LH}$ could mediate innate behaviors through specifically tuned neurons we did not observe, by responding preferentially to categories of odors we did not test, or through another mechanism such as the combinatorial coding scheme used by PNs (Laurent, 2002).

The great diversity of LHNs we observed suggests that the LH may perform other sensory functions, though. Our observations lead us to speculate about three possible roles: representing gen- eral odor properties such as intensity (Fig. 5), beginning bilateral olfactory integration (Fig. 6A), and participating in multimodal integration (Fig. 6B,E). These possible functions could all contribute to unlearned olfactory tasks such as odor tracking. Hopfield (1995) proposed that phase coding may allow extremely fast decoding of stimulus intensity by downstream cells. Class C3 neurons, which contain information about the odor concentration in their spike phases (Fig. 5A) and show bilateral projections (Fig. 6A1), could assist in bilateral integration in the locust by allowing rapid comparison of odor intensity across the midline, a feature potentially important for odor tracking in some animals (Martin, 1965; Rajan et al., 2006).

Previous work in Drosophila identified neurons connecting the LH with different parts of the brain, including those serving other senses (Tanaka et al., 2004, 2008; Jefferis et al., 2007; Ruta et al., 2010). We found several neurons that responded to both visual and olfactory stimuli (Fig. 6E), providing direct evidence for multimodal responses in the LH. Behavioral studies have shown that visual input contributes to odor tracking (Frye et al., 2003). Thus, in addition to possibly integrating input from the two antennae, neurons of the LH may mediate integration of olfactory and visual cues for odor-tracking behaviors in insects (Duistermars and Frye, 2010). Our results provide specific neuronal targets, from the variety of LHN classes, for further investigation into mechanisms underlying odor tracking. If the term "innate behaviors" can be used broadly to encompass all memory-independent behaviors such as odor tracking regardless of odor identity, our results support the role of $\mathrm{LH}$ in these behaviors.

\section{References}

Assisi C, Stopfer M, Laurent G, Bazhenov M (2007) Adaptive regulation of sparseness by feedforward inhibition. Nat Neurosci 10:1176-1184.

Bicker G, Schäfer S, Kingan TG (1985) Mushroom body feedback interneurones in the honeybee show GABA-like immunoreactivity. Brain Res 360:394-397.

Brown SL, Joseph J, Stopfer M (2005) Encoding a temporally structured stimulus with a temporally structured neural representation. Nat Neurosci 8:1568-1576.

Carey AF, Carlson JR (2011) Insect olfaction from model systems to disease control. Proc Natl Acad Sci U S A 108:12987-12995.

Cassenaer S, Laurent G (2007) Hebbian STDP in mushroom bodies facilitates the synchronous flow of olfactory information in locusts. Nature 448:709-713.

Cassenaer S, Laurent G (2012) Conditional modulation of spike-timingdependent plasticity for olfactory learning. Nature 482:47-52.

Daly KC, Durtschi ML, Smith BH (2001) Olfactory-based discrimination learning in the moth, Manduca sexta. J Insect Physiol 47:375-384.

Datta SR, Vasconcelos ML, Ruta V, Luo S, Wong A, Demir E, Flores J, Balonze K, Dickson BJ, Axel R (2008) The Drosophila pheromone cVA activates a sexually dimorphic neural circuit. Nature 452:473-477.

Davis RL (2011) Traces of Drosophila memory. Neuron 70:8-19.

de Belle JS, Heisenberg M (1994) Associative odor learning in Drosophila abolished by chemical ablation of mushroom bodies. Science 263:692-695.

Demmer H, Kloppenburg P (2009) Intrinsic membrane properties and inhibitory synaptic input of kenyon cells as mechanisms for sparse coding? J Neurophysiol 102:1538-1550.

Duistermars BJ, Frye MA (2010) Multisensory integration for odor tracking by flying Drosophila: behavior, circuits and speculation. Commun Integr Biol 3:60-63.

Ernst KD, Boeckh J, Boeckh V (1977) A neuroanatomical study on the organization of the central antennal pathways in insects. Cell Tissue Res 176:285-306.

Finelli LA, Haney S, Bazhenov M, Stopfer M, Sejnowski TJ (2008) Synaptic learning rules and sparse coding in a model sensory system. PLoS Comput Biol 4:e1000062.

Frye MA, Tarsitano M, Dickinson MH (2003) Odor localization requires 
visual feedback during free flight in Drosophila melanogaster. J Exp Biol 206:843-855.

Grosjean Y, Rytz R, Farine JP, Abuin L, Cortot J, Jefferis GS, Benton R (2011) An olfactory receptor for food-derived odours promotes male courtship in Drosophila. Nature 478:236-240.

Hansson BS ed. (1999) Insect olfaction. Berlin: Springer.

Heimbeck G, Bugnon V, Gendre N, Keller A, Stocker RF (2001) A central neural circuit for experience-independent olfactory and courtship behavior in Drosophila melanogaster. Proc Natl Acad Sci U S A 98:15336-15341.

Homberg U, Kingan TG, Hildebrand JG (1987) Immunocytochemistry of GABA in the brain and suboesophageal ganglion of Manduca sexta. Cell Tissue Res 248:1-24.

Homberg U, Christensen TA, Hildebrand JG (1989) Structure and function of the deutocerebrum in insects. Ann Rev Entomol 34:477-501.

Honegger KS, Campbell RA, Turner GC (2011) Cellular-resolution population imaging reveals robust sparse coding in the Drosophila mushroom body. J Neurosci 31:11772-11785.

Hopfield JJ (1995) Pattern recognition computation using action potential timing for stimulus representation. Nature 376:33-36.

Ito I, Ong RC, Raman B, Stopfer M (2008) Sparse odor representation and olfactory learning. Nat Neurosci 11:1177-1184.

Jefferis GS, Potter CJ, Chan AM, Marin EC, Rohlfing T, Maurer CR Jr, Luo L (2007) Comprehensive maps of Drosophila higher olfactory centers: spatially segregated fruit and pheromone representation. Cell 128:1187-1203.

Jortner RA, Farivar SS, Laurent G (2007) A simple connectivity scheme for sparse coding in an olfactory system. J Neurosci 27:1659-1669.

Kazama H, Wilson RI (2009) Origins of correlated activity in an olfactory circuit. Nat Neurosci 12:1136-1144.

Keene AC, Waddell S (2007) Drosophila olfactory memory: single genes to complex neural circuits. Nat Rev Neurosci 8:341-354.

Kido A, Ito K (2002) Mushroom bodies are not required for courtship behavior by normal and sexually mosaic Drosophila. J Neurobiol 52:302-311.

Larimer P, Strowbridge BW (2008) Nonrandom local circuits in the dentate gyrus. J Neurosci 28:12212-12223.

Laurent G (2002) Olfactory network dynamics and the coding of multidimensional signals. Nat Rev Neurosci 3:884-895.

Laurent G, Naraghi M (1994) Odorant-induced oscillations in the mushroom bodies of the locust. J Neurosci 14:2993-3004.

Leitch B, Laurent G (1996) GABAergic synapses in the antennal lobe and mushroom body of the locust olfactory system. J Comp Neurol 372:487-514.

Lin HH, Lai JS, Chin AL, Chen YC, Chiang AS (2007) A map of olfactory representation in the Drosophila mushroom body. Cell 128:1205-1217.

Liu X, Davis RL (2009) The GABAergic anterior paired lateral neuron suppresses and is suppressed by olfactory learning. Nat Neurosci 12:53-59.

Louis M, Huber T, Benton R, Sakmar TP, Vosshall LB (2008) Bilateral olfactory sensory input enhances chemotaxis behavior. Nat Neurosci 11:187-199.

Luo SX, Axel R, Abbott LF (2010) Generating sparse and selective thirdorder responses in the olfactory system of the fly. Proc Natl Acad Sci U S A 107:10713-10718.

MacLeod K, Bäcker A, Laurent G (1998) Who reads temporal information contained across synchronized and oscillatory spike trains? Nature 395:693-698.

Marin EC, Jefferis GS, Komiyama T, Zhu H, Luo L (2002) Representation of the glomerular olfactory map in the Drosophila brain. Cell 109:243-255.

Martin H (1965) Osmotropotaxis in the honey-bee. Nature 208:59-63.

Masse NY, Turner GC, Jefferis GS (2009) Olfactory information processing in Drosophila. Curr Biol 19:R700-R713.

Papadopoulou M, Cassenaer S, Nowotny T, Laurent G (2011) Normalization for sparse encoding of odors by a wide-field interneuron. Science 332:721-725.

Perez-Orive J, Mazor O, Turner GC, Cassenaer S, Wilson RI, Laurent G
(2002) Oscillations and sparsening of odor representations in the mushroom body. Science 297:359-365.

Perez-Orive J, Bazhenov M, Laurent G (2004) Intrinsic and circuit properties favor coincidence detection for decoding oscillatory input. J Neurosci 24:6037-6047.

Pitman JL, Huetteroth W, Burke CJ, Krashes MJ, Lai SL, Lee T, Waddell S (2011) A Pair of inhibitory neurons are required to sustain labile memory in the Drosophila mushroom body. Curr Biol 21:855-861.

Poo C, Isaacson JS (2009) Odor representations in olfactory cortex: "sparse" coding, global inhibition, and oscillations. Neuron 62:850-861.

Porter J, Craven B, Khan RM, Chang SJ, Kang I, Judkewitz B, Judkewicz B, Volpe J, Settles G, Sobel N (2007) Mechanisms of scent-tracking in humans. Nat Neurosci 10:27-29.

Rajan R, Clement JP, Bhalla US (2006) Rats smell in stereo. Science 311:666-670.

Ruta V, Datta SR, Vasconcelos ML, Freeland J, Looger LL, Axel R (2010) A dimorphic pheromone circuit in Drosophila from sensory input to descending output. Nature 468:686-690.

Stocker RF, Lienhard MC, Borst A, Fischbach KF (1990) Neuronal architecture of the antennal lobe in Drosophila melanogaster. Cell Tissue Res 262:9-34.

Stokes CC, Isaacson JS (2010) From dendrite to soma: dynamic routing of inhibition by complementary interneuron microcircuits in olfactory cortex. Neuron 67:452-465.

Stopfer M, Laurent G (1999) Short-term memory in olfactory network dynamics. Nature 402:664-668.

Stopfer M, Jayaraman V, Laurent G (2003) Intensity versus identity coding in an olfactory system. Neuron 39:991-1004.

Tanaka NK, Awasaki T, Shimada T, Ito K (2004) Integration of chemosensory pathways in the Drosophila second-order olfactory centers. Curr Biol 14:449-457.

Tanaka NK, Tanimoto H, Ito K (2008) Neuronal assemblies of the Drosophila mushroom body. J Comp Neurol 508:711-755.

Touhara K, Vosshall LB (2009) Sensing odorants and pheromones with chemosensory receptors. Ann Rev Physiol 71:307-332.

Turner GC, Bazhenov M, Laurent G (2008) Olfactory representations by Drosophila mushroom body neurons. J Neurophysiol 99:734-746.

Vickers N, Baker T (1996) Latencies of behavioral response to interception of filaments of sex pheromones and clean air influence flight track shape in Heliothis virescens (F.) males. J Comp Physiol A Neuroethol Sens Neural Behav Physiol 178:831-847.

Wang Y, Chiang AS, Xia S, Kitamoto T, Tully T, Zhong Y (2003) Blockade of neurotransmission in Drosophila mushroom bodies impairs odor attraction, but not repulsion. Curr Biol 13:1900-1904.

Wehr M, Laurent G (1996) Odour encoding by temporal sequences of firing in oscillating neural assemblies. Nature 384:162-166.

Wong AM, Wang JW, Axel R (2002) Spatial representation of the glomerular map in the Drosophila protocerebrum. Cell 109:229-241.

Wu CL, Shih MF, Lai JS, Yang HT, Turner GC, Chen L, Chiang AS (2011) Heterotypic gap junctions between two neurons in the Drosophila brain are critical for memory. Curr Biol 21:848-854.

Xia S, Tully T (2007) Segregation of odor identity and intensity during odor discrimination in Drosophila mushroom body. PLoS Biol 5:e264.

Yamagata N, Mizunami M (2010) Spatial representation of alarm pheromone information in a secondary olfactory centre in the ant brain. Proc $\mathrm{R}$ Soc Lond B Biol Sci 277:2465-2474.

Yamazaki Y, Nishikawa M, Mizunami M (1998) Three classes of GABA-like immunoreactive neurons in the mushroom body of the cockroach. Brain Res 788:80-86.

Yu JY, Kanai MI, Demir E, Jefferis GS, Dickson BJ (2010) Cellular organization of the neural circuit that drives Drosophila courtship behavior. Curr Biol 20:1602-1614. 